Document downloaded from:

http://hdl.handle.net/10251/165515

This paper must be cited as:

Gisbert Soler, V.; Jiménez-Serrano, S.; Roses-Albert, E.; Rodrigo Bort, M. (2020). Atrial location optimization by electrical measures for Electrocardiographic Imaging. Computers in Biology and Medicine. 127:1-8. https://doi.org/10.1016/j.compbiomed.2020.104031

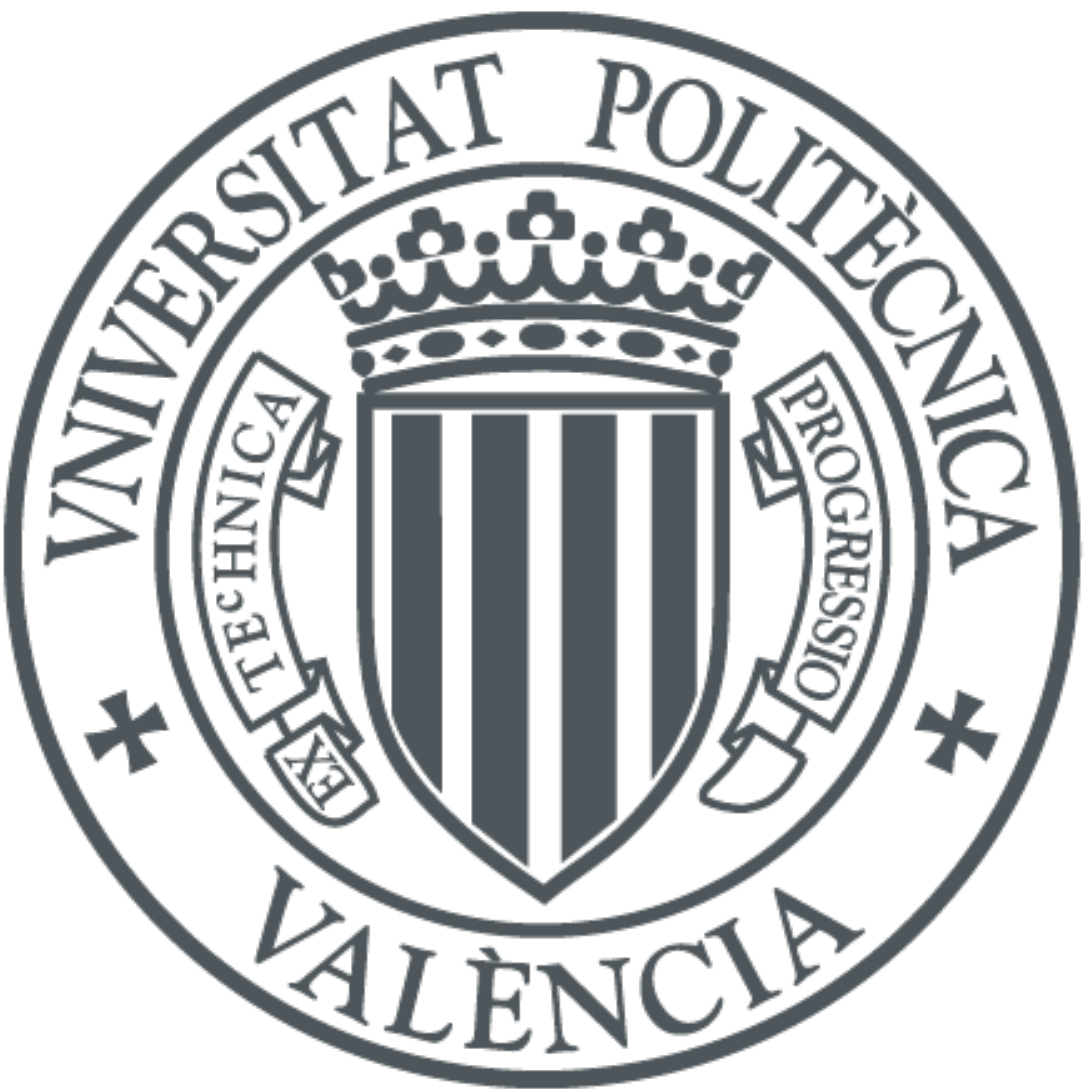

The final publication is available at

https://doi.org/10.1016/j.compbiomed.2020.104031

Copyright Elsevier

Additional Information 


\title{
Atrial Location Optimization by Electrical Measures for Electrocardiographic Imaging
}

\author{
Víctor Gisbert ${ }^{1}$, Santiago Jiménez-Serrano ${ }^{1}$, Eduardo Roses-Albert ${ }^{1-2}$ and Miguel \\ Rodrigo ${ }^{1}$ \\ ${ }^{1}$ ITACA Institute, Universitat Politècnica de València, Valencia, Spain. \\ ${ }^{2}$ Proteu Tecnología Aplicada Coop V
}

Running head: Electrical Atrial Location on ECGI

Corresponding author:

Miguel Rodrigo (mirodbor@teleco.upv.es)

ITACA Institute, Universitat Politècnica de València

Telephone/FAX: +34987 7700

Cami de Vera s/n, 46022, Valencia, Spain

\section{Financial support}

This work was supported in part by: Generalitat Valenciana Grants [APOSTD/2017] and projects [GVA/2018/103]; Nvidia Corporation with GPU QUADRO P6000 donation.

\section{Conflict of interest}

M. Rodrigo is shareholders of Corify Health Care S.L. 


\title{
ABSTRACT AND KEY TERMS
}

\begin{abstract}
Background: The Electrocardiographic Imaging (ECGI) technique, used to non-invasively reconstruct the epicardial electrical activity, requires an accurate model of the atria and torso anatomy. Here we evaluate a new automatic methodology able to locate the atrial anatomy within the torso based on an intrinsic electrical parameter of the ECGI solution.

Methods: In 28 realistic simulations of the atrial electrical activity, we randomly displaced the atrial anatomy for $\pm 2.5 \mathrm{~cm}$ and \pm 30 degrees on each axis. An automatic optimization method based on the L-curve curvature was used to estimate the original position using exclusively non-invasive data.

Results: The automatic optimization algorithm located the atrial anatomy with a deviation of $0.5 \pm$ $0.5 \mathrm{~cm}$ in position and $16.0 \pm 10.7^{\circ}$ in orientation. With these approximate locations, the obtained electrophysiological maps reduced the average error in atrial rate measures from $1.1 \pm 1.1 \mathrm{~Hz}$ to $0.5 \pm 1.0 \mathrm{~Hz}$ and in the phase singularity position from $7.2 \pm 4.0 \mathrm{~cm}$ to $1.6 \pm 1.7 \mathrm{~cm}(p<0.01)$.

Conclusions: This proposed automatic optimization may help to solve spatial inaccuracies provoked by cardiac motion or respiration, as well as to use ECGI on torso and atrial anatomies from different medical image systems.
\end{abstract}

Keywords: Inverse problem; L-curve curvature; Electrophysiology; Mapping; Dominant Frequency; Phase analysis; Reentry; Rotor

List of Abbreviations:

AF: Atrial fibrillation

DF: Dominant Frequency

ECG: Electrocardiogram

ECGI: Electrocardiographic Imaging

EGM: Electrogram (Intracardiac)

icEGM: inverse-computed Electrogram 


\section{INTRODUCTION}

The use of non-invasive techniques for mapping the cardiac activity such as Electrocardiographic Imaging (ECGI) provides a panoramic view of the electrical activity during heart diseases and sinus rhythm. The ECGI has been exploited in the study of both ventricular [1-2], and atrial arrhythmias [3-6]. In Atrial Fibrillation (AF), the most frequent arrhythmia in clinical practice, ECGI technology has been used to non-invasively identify the atrial regions responsible of the arrhythmia maintenance, in form of reentrant activity or rapidly activated regions [3-4].

The ECGI reconstruction of the cardiac potentials is conducted through the solution of the so-called inverse-problem, using the surface electrical activity and the torso and heart anatomy [7]. However, an accurate three-dimensional model of the torso and heart requires the use of medical imaging techniques, such as MRI or CT, whose images must be manual or semi-automatically segmented. Nowadays, image reconstruction from MRI/CT limits the use of the ECGI in routine clinical practice, as these image techniques are commonly used only on the minor AF sub-population referred for surgical procedures.

In prior studies we showed how an intrinsic parameter involved in the electrical ECGI reconstruction, the curvature of the L-curve, allows to solve inaccuracies in the heart anatomy location [8]. Due to the availability of atrial and torso geometries from different recording methods such as photogrammetry, echocardiography, anatomical atlases, etc., we hypothesize that this L-curve approach could be used to align the torso and atrial geometries obtained from different imaging methods and intrinsically in different coordinate spaces. Moreover, this technique may help also to diminish the spatial artifacts present in MRI/CT segmentations, such as those provoked by patient motion or respiration.

However, the heart anatomy location within the torso is a spatial problem with 6 degrees of freedom ( 3 axes of displacement and rotation), and its solution by an optimization algorithm is not straightforward. This study presents an optimization algorithm able to automatically solve the atrial location and orientation based exclusively on surface electrical measures, using a discrete search optimization algorithm. Departing from our previous work in which we showed the dependence of the atrial location and the L-curve curvature [8], this manuscript proposes an optimization algorithm to automatically locate the atrial anatomy, as well as the validation of this location algorithm using clinical AF markers. 


\section{MATERIALS AND METHODS}

\section{Realistic simulations of the atrial electrical activity}

A 3D model of the atrial anatomy composed by 284,578 nodes and $1,353,783$ tetrahedra was used to simulate the atrial electrical activity (673 $\pm 130 \mu \mathrm{m}$ inter-node) [9]. Each node was simulated as a single atrial cell using the cellular model described by Koivumaki et al. [10] and solved using an adaptive algorithm described in [11]. Heterogeneity in the electrophysiological properties of atrial myocardium was introduced in form of changes in ion currents and distribution of fibrosis to generate AF episodes maintained by reentrant activity and fibrillatory activity with non-uniform propagation patterns and different shape and extent of the dominant region [12]. 28 different AF episodes of 10 seconds-length driven by a single and spatially-stable rotor in different locations were used in this work. For each simulation, a uniform mesh of 2048 nodes ( $5.3 \pm 3.2 \mathrm{~mm}$ inter-node) of unipolar electrograms (EGM) was calculated at $1 \mathrm{~mm}$ from the surface of the epicardium under the assumption of a homogeneous, unlimited and quasi-static conductive medium adding all effective dipole contributions over the entire model. The computed electrograms were stored for processing at a sampling frequency of $500 \mathrm{~Hz}$ [12].

The ECG potentials at the torso surface were calculated by solving the Boundary Elements Method $[4,7]$ in a mesh formed by 771 nodes and 1538 triangular patches (3.8 $\pm 1.0 \mathrm{~cm}$ inter-node) [13]. White Gaussian noise was added to the synthetic ECG signals at a signal-to-noise ratio of $20 \mathrm{~dB}$ [12].

\section{Inverse problem and L-Curve}

The ECGI solution consists on the reconstruction of the epicardial electrical activity through the inverse-problem resolution. Contrary to the forward problem, which allows to calculate the surface electrical activity from the epicardial activity, the inverse problem calculates the inverse-computed Electrograms (icEGM) from the surface electrical activity (ECG) using:

$$
\operatorname{icEGM}(\lambda)=M^{-1} \cdot E C G
$$

where $M$ is the forward matrix describing the projection of the surface electrical activity from the epicardial potentials through their anatomy. Since $M$ is defined for the forward problem, its inverse matrix cannot be calculated in terms of classical linear algebra. The linear equation system can be solved using the zero-order Tikhonov method, which substitutes the inverse of the $M$ matrix by an invertible matrix $[4,7]$ :

$$
\operatorname{icEGM}(\lambda)=\left(M^{t} \cdot M+\lambda \cdot I^{t} \cdot I\right)^{-1} \cdot M^{t} \cdot E C G
$$

where $I$ is the identity matrix and $\lambda$ is a constant regularization parameter that does not depend on time. The optimal regularization parameter is selected according to the L-curve method, which allows to compensate the errors in the solution (residual norm: $\log \left(\|M \cdot i c E G M(\lambda)-E C G\|^{2}\right)$ ) with the numerical errors provoked by the matrix inversion (solution norm: $\log \left(\|i c E G M(\lambda)\|^{2}\right)$ ) by minimizing the expression $\|M \cdot i c E G M(\lambda)-E C G\|^{2}+\lambda^{2}\|i c E G M(\lambda)\|^{2}$, being the operator $(\|\cdot\|)$ the Frobenius norm. This was carried out by the L-cuve method, a graphical method which selects the regularization parameter $\lambda$ that constitutes the corner in the $X-Y$ graph between the residual and the solution log norms. The corner of the curve $L$ is then defined as the first point of maximum curvature according to: 


$$
\operatorname{curvature}(\lambda)=\frac{\frac{d x}{d \lambda} \cdot \frac{d^{2} y}{d \lambda^{2}}-\frac{d y}{d \lambda} \cdot \frac{d^{2} x}{d \lambda^{2}}}{\left[\left(\frac{d x}{d \lambda}\right)^{2}+\left(\frac{d y}{d \lambda}\right)^{2}\right]^{\frac{3}{2}}}
$$

where $x$ is the logarithm of the residual norm $\left(\log \|M \cdot i c E G M(\lambda)-E C G\|^{2}\right)$ and $y$ the logarithm of the solution norm $\left(\log \|i c E G M(\lambda)\|^{2}\right)$. The optimal regularization parameter was chosen at the first maximum value of the curvature $[4,7,8]$. Previous studies of our group showed that the maximal curvature value of the L-curve is a good estimator of the proximity of the atrial position and orientation from its natural location [8].

\section{Optimization algorithm}

In order to automatically locate the atrial anatomy within the torso based on the L-curve curvature a classical optimization algorithm was used: Pattern Search [14-15], which belongs to the family of numerical methods not based on gradient calculation. This algorithm is used for convergence of maximums and performs a discrete search with variable meshing. In our case, the optimization algorithm explores a 6-dimension space ( 3 axes of displacement and rotation) looking for the 6D point (corresponding to a heart position and orientation) whose ECGI solution gives the maximal Lcurve curvature. Our hypothesis departs from the basis that the location in which the heart was located when the ECGI recordings are performed produces the maximal curvature of the L-curve, compared to altered locations [8]. Therefore, here the optimization algorithm is used to minimize the spatial artifacts present in a supposed altered heart location by optimizing those 6 parameters (position and orientation) to obtain the maximal L-curve-curvature.

The quest starts with a fixed meshing interval and performs an initial search in 12 points with an individual increment in each of the 6 axes of freedom ( 3 for displacement and 3 for rotation). Then, it solves the inverse problem and looks for the maximal value of the L-curve in these 13 points: the current point and 12 independent increments ( \pm meshing interval) in each axis. Whether the maximal curvature is found in a position different from the current point, the algorithm displaces to that position and the meshing interval is doubled. Otherwise, the algorithm remains in the same location and the meshing interval is reduced by $50 \%$, until changes between iterations are below a tolerance (see Fig. 1C).

\section{Electrophysiological markers for atrial fibrillation}

Due to the regularization process of the inverse solution, the icEGMs signals reconstructed on AF episodes have been reported to depict incomplete information regarding local electrical activation $[12,16]$, and are hard to be visually interpreted. Therefore, icEGM signals are commonly postprocessed in order to measure known electrophysiological markers related with the AF driver positions, target of the ablation procedures and objective of the ECGI mapping in AF.

From classic electrophysiological parameters, this paper will evaluate the local temporal evolution of the icEGMs traces by using measures of signal correlation. For post-processing markers, this work will evaluate the activation frequency maps and the reentrant activity detected by phase analysis. These are two of the main techniques used in clinical practice for AF mapping, as they allow to identify the fastest-activated and the reentry-activated regions, both features suspected to be markers of the atrial regions responsible of AF maintenance [17-18]. The activation frequency maps were calculated through the Dominant Frequency (DF) algorithm, which obtains the maximal 
spectral contribution in the frequency domain after applying the Welch periodogram [17]. For the reentrant activity detection, the atrial icEGMs were pre-filtered using a narrow band-pass filtering centered at the highest DF and phase signals were obtained through the Hilbert Transform. Stable phase singularities were identified in these phase maps as described in [19]. Finally, the reentrant activity in 4 seconds-length episodes was summarized in a phase singularity probability map, where the amount of reentrant activity occurrences were depicted for each atrial region. The node with the highest reentrant contribution was considered as the AF reentrant driver position.

\section{Description of the Experiments}

The EGMs simulated at the original position for the 28 different AF episodes were used to calculate their corresponding surface ECGs through the forward problem. The same atrial anatomy was used to simulate all $28 \mathrm{AF}$ episodes. This atrial anatomy was then displaced and rotated from its original position by $\pm 2.5 \mathrm{~cm}$ and $\pm 30^{\circ}$ in all 3 displacement and rotation axes randomly. The original surface ECG signals were then used to run the location algorithm based on the L-curve. An initial meshing interval of 2 ( $\mathrm{cm}$ or degrees) was used and the tolerance was set to 0.125 (cm or degrees).

The optimization algorithm was run twice per episode, with 2 different initial positions, in order to check if the same final positions (global maximum) were given by both initial points. Once obtained the final position by the optimization algorithm, the electrophysiological markers described in the previous section were calculated for the icEGM signals at the original, initial and final position of the algorithm. Finally, these markers were compared between the original and initial/final positions, in order to test the clinical usefulness of the proposed solution.

The optimization algorithm and the experiments were coded on Matlab (Natick, USA). The most computational-expensive parts of the optimization algorithm (forward matrix calculation and matrix inversion from the Tikhonov regularization) were accelerated by parallel computing on Graphic Processor Unit (GPU) using CUDA code and linked to Matlab through a dedicated dynamic-link library (DLL). The number of iterations of each experiment as well as the computation time was measured on a 8-cores CPU (i7-7700, 32 RAM GB, $3.60 \mathrm{GHz}$ ) with a dedicated GPU (Nvidia P6000, 24 RAM GB, 3840 cores).

Results were expressed as average \pm standard deviation when standard normal distribution was observed (using Kolmogorov-Smirnov test), or as median [interquartile range] otherwise. Statistical significance was checked using T-student test for variables with standard normal distribution or using Wilcoxon rank sum test otherwise. Statistical significance was considered when $p<0.01$. 


\section{RESULTS}

\section{Optimization algorithm for location}

In Figure 1 an illustrative example of the optimization process is depicted. In panel A, the torso mesh (green) and the atrial anatomy at its original position (red) are shown. Panel B shows the path followed by the algorithm in one of the optimization processes from the initial position $(2.5,2.5,-$ $2.5)$ to the final position $(0,0,0.5)$, and compared with the original position $(0,0,0)$. The algorithm found the maximal L-curve curvature $0.5 \mathrm{~cm}$ away from the original position. While the deviation in orientation in the starting position was $-30 \circ$ in the $X$ axis, $30^{\circ}$ in the $Y$ axis and $-30 \circ$ in the $Z$ axis, the angular deviation at the final position from the actual orientation was $2^{\circ}$ in the $X$ axis, $-18^{\circ}$ in the $Y$ axis and $-2^{\circ}$ in the $Z$ axis.
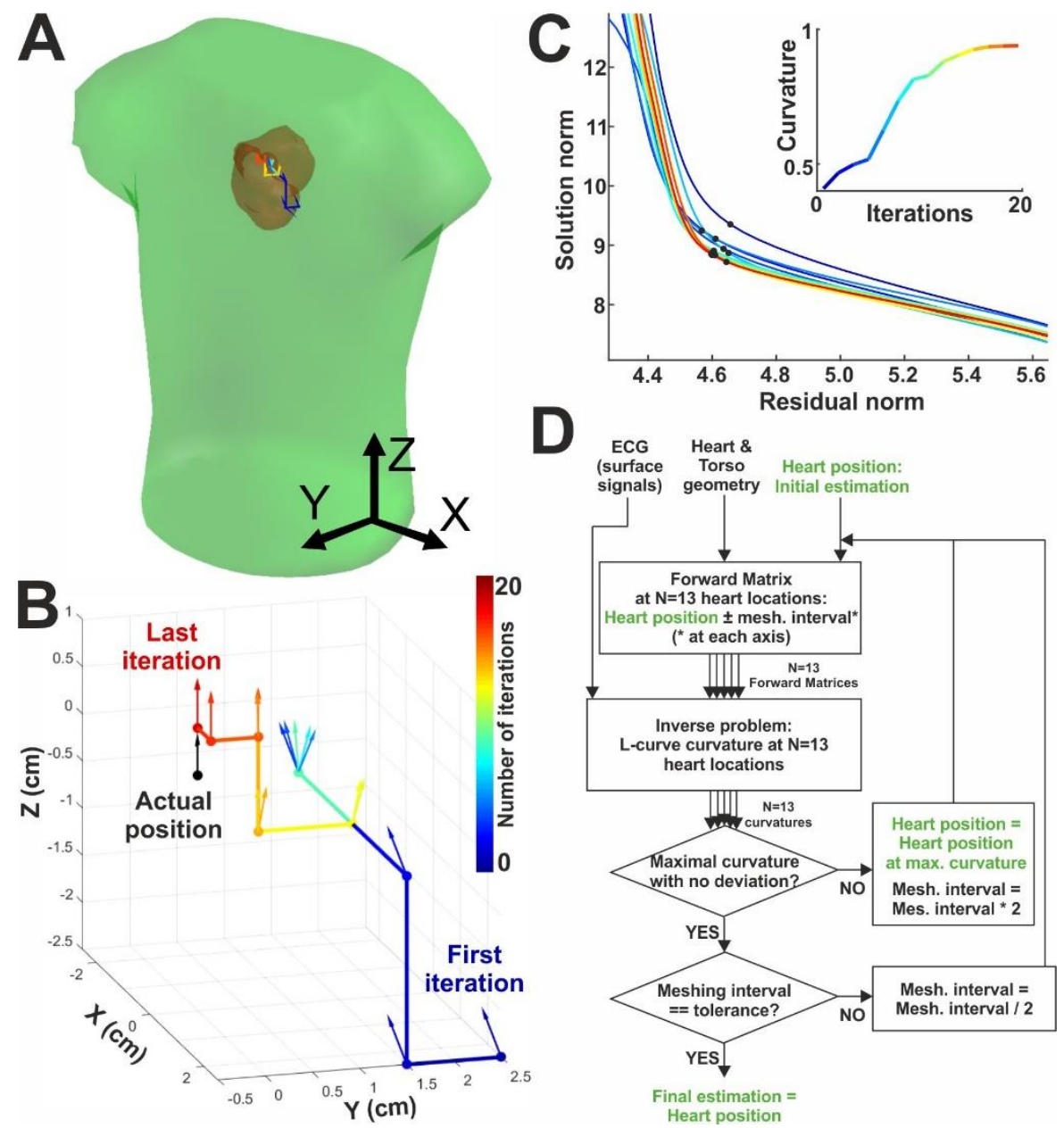

Fig. 1. Illustrative example. A. Torso geometry (green) and atrial geometry (red). B. Example of the localization algorithm using $[2.5,2.5,-2.5]$ as starting point and $[0,0,0.5]$ as final point respect the actual location $[0,0,0]$. C. L-curves and their curvature at the maximal curvature point (black dot) for the example in Panel B, color-coded according to the optimization algorithm iteration. $D$. Flowchart of the optimization algorithm

For the entire database, composed by the 28 different simulations in which the optimization algorithm was run twice (56 tests), the average error between the original and the final position is shown in Figure 2, for an initial error of $4.3 \mathrm{~cm}$ and $52^{\circ}$ in random directions. The final location for the 56 test was obtained after 17 [9 - 33] iterations in 748 [434 - 1686] seconds. For displacements 
(Panel A), the location algorithm provided a deviation between the actual and the final location of $0.2 \pm 0.2 \mathrm{~cm}$ in the $X$ axis, $0.2 \pm 0.3 \mathrm{~cm}$ for the $Y$ axis and $0.4 \pm 0.3 \mathrm{~cm}$ for the $Z$ axis, resulting in an absolute error of $0.5 \pm 0.5 \mathrm{~cm}$ in terms of Euclidean distance. For angular deviation (Panel B) we observed a deviation of $4.8 \pm 3.4^{\circ}$ for the $X$ axis, $14.5 \pm 9.7^{\circ}$ for the $Y$ axis and $4.8 \pm 3.0^{\circ}$ for the $Z$ axis, obtaining an absolute deviation of $16.0 \pm 10.7^{\circ}$.

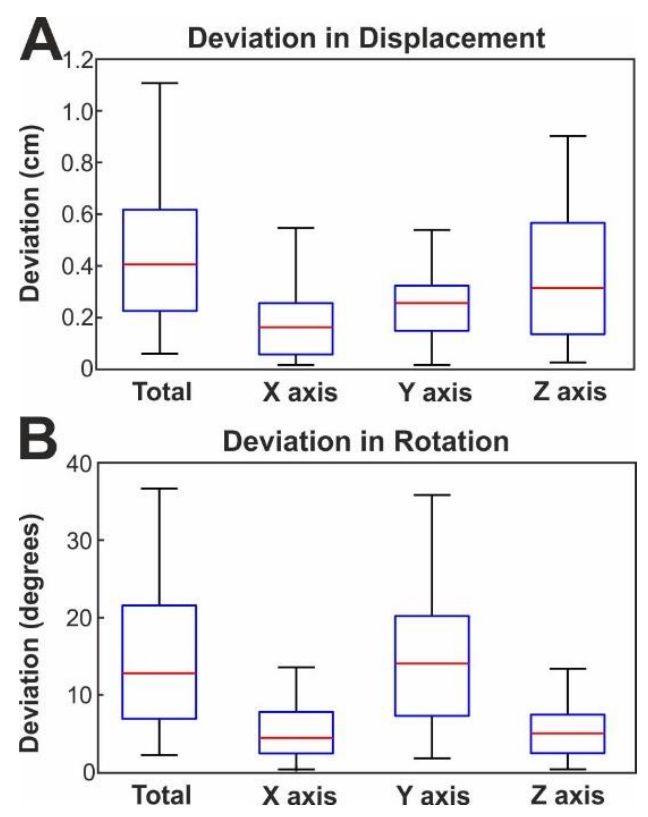

Fig. 2. Location deviations for displacement (A) and rotation (B).

In $82 \%$ of cases (23/28 simulations), the optimization solutions for the 2 different starting points of the same simulation gave the same final position (difference lower than $0.2 \mathrm{~cm}$ ), with an average Euclidean distance between both solutions of $0.3 \pm 0.2 \mathrm{~cm}$ along the entire database. In $68 \%$ of cases $(19 / 28)$ the final solution gave the same orientation for both cases (difference lower than 0.2), with a relative error between orientations of same simulation of $0.5 \pm 0.3 \circ$ along the entire database. In $54 \%$ of cases $(14 / 28)$ both position and orientation matched between solutions, indicating that the optimization algorithm provided the same or similar global maximal solution from different starting points.

\section{Inverse-computed EGM maps and traces}

In Figure 3 the inverse-reconstructed voltage maps obtained at the actual position, initial and final iteration of the algorithm are depicted, at the instant in which the spatial correlation is higher between the actual and the final potential distribution. On this example, same as Fig. 1, the deviation on the final position was $0.5 \mathrm{~cm}$ and 18 . Potential distributions at the actual location and at the first iteration presented important differences: electrical dipoles at positions \#1 and \#2 in the actual position are displaced to lower positions on the first iteration. The reconstructed map at the final position preserves the original potential distribution, as the location of maximum and minimum potential appear at similar locations. 


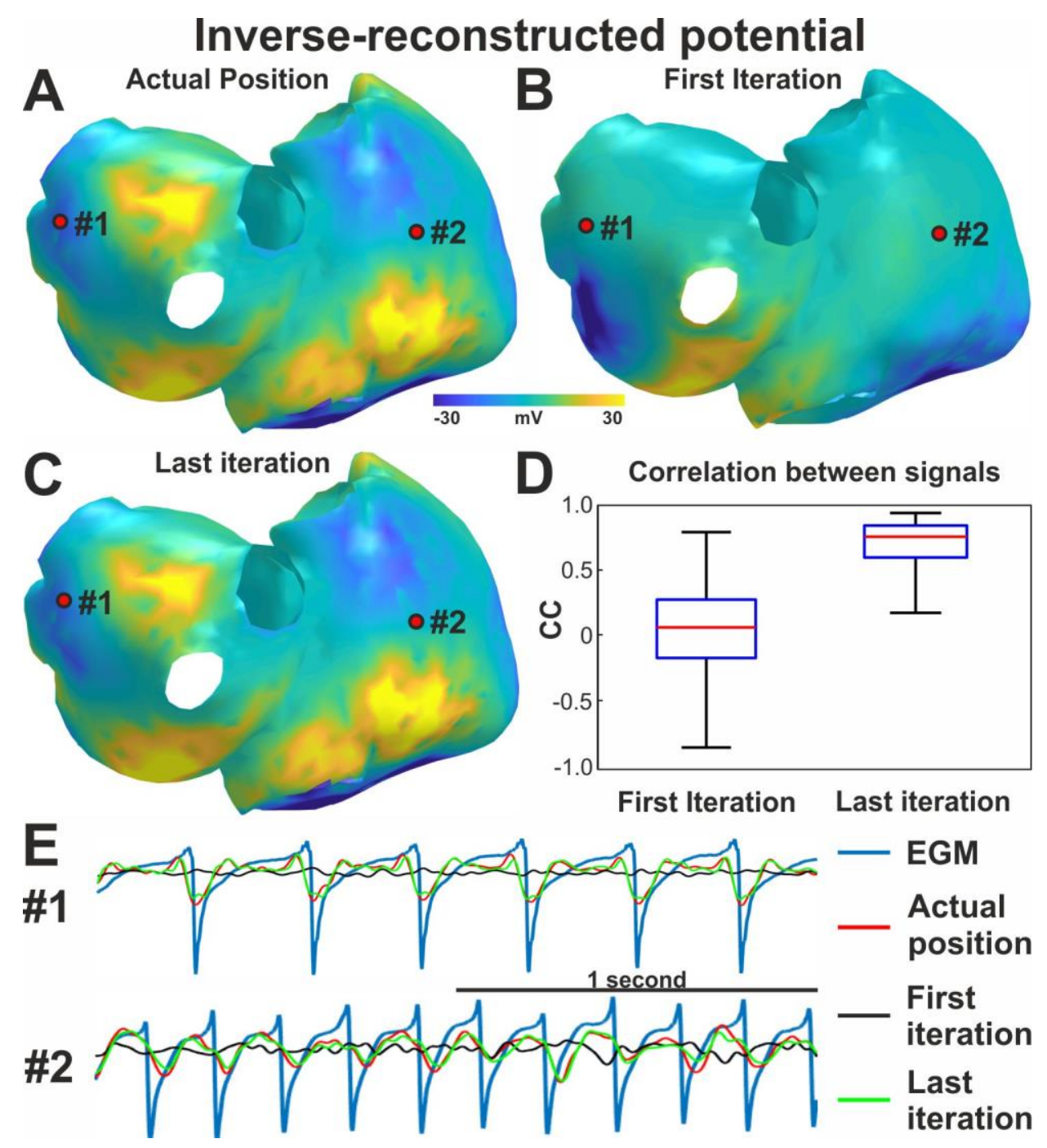

Fig. 3. Reconstruction of the epicardial potential. Potential distribution maps at the actual position (A), first iteration (B) and last iteration (C). D. Correlation Coefficient (CC) between the potential signals at the actual vs. at the first (left) or last (right) iteration. E. Example of 2 reconstructed potential traces at positions marked in panels $A-C$.

The corresponding EGM and inverse-computed EGM (icEGM) traces for the 2 points marked in Panels A-C are depicted in Panel E. All inverse-computed signals had lower amplitudes than the starting EGM, due to the regularization process, but the icEGM traces at the original and final positions showed an activation sequence similar to the starting EGM. Nevertheless, the icEGM trace obtained at the initial point (in black) did not show clear activations and they were unrelated with the starting EGM. The agreement between individual signal traces can be observed in Panel D, where the correlation coefficients between the icEGM at the actual position and the icEGMs at the first and last iterations of the location algorithm were calculated for each individual atrial node. We obtained a moderate correlation coefficient $(0.74 \pm 0.17)$ between the actual and last positions, compared to the obtained between the actual and initial positions $(0.02 \pm 0.32, p<0.01)$. However, as the spatial distribution of the electrical potential was relatively well maintained at the final iteration compared with the actual position, their average spatial CC values were higher (0.98 \pm 
0.01) than the CC across temporal signals. Spatial CC between initial and actual position was also higher than for temporal CC $(0.20 \pm 0.18, p<0.01)$ although still showing low spatial correlation.

\section{Activation frequency and reentrant activity maps}

Figure 4 shows the Dominant Frequency (DF) maps obtained at the actual, first and last position for the example on Figure 3. At the original position, the fastest activated region was present at the RA lateral wall and activated around $5.5 \mathrm{~Hz}$, whereas the rest of the atria was activated at lower frequencies $(3 \mathrm{~Hz})$. The final DF distribution resembled the original, and the fastest region can be identified as a region with similar shape and location and activated at the same frequency $(5.5 \mathrm{~Hz})$. However, the DF map at the initial iteration resulted unable to reconstruct the starting DF distribution, showing small regions at $5.5 \mathrm{~Hz}$ distributed in areas different to the original fastest site.

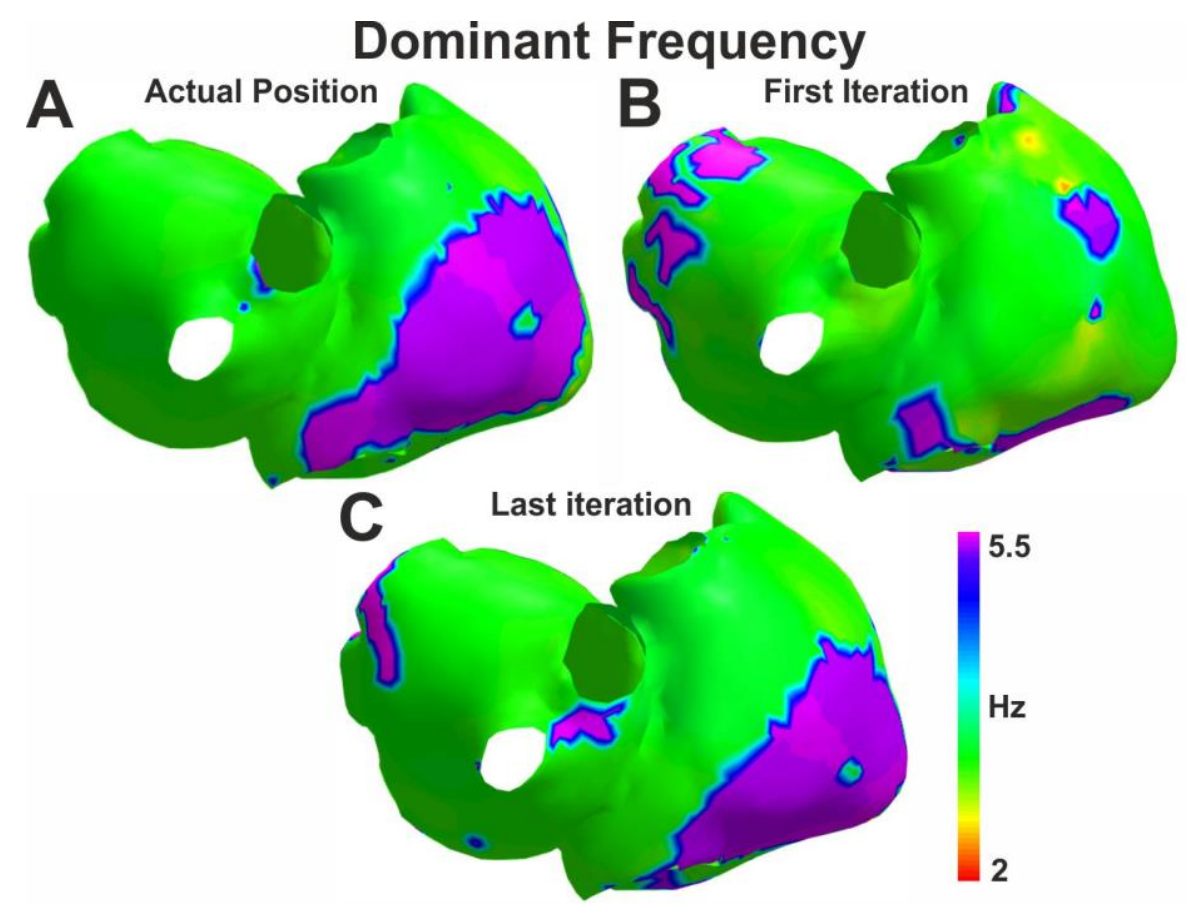

Fig. 4. Reconstruction of activation rate maps through Dominant Frequency analysis at the actual position (A), first iteration (B) and last iteration (C).

Figure 5 shows an example of the reentrant activity analysis. In this example, with a final deviation of $0.7 \mathrm{~cm}$ and $12^{\circ}$, the $A F$ episode was sustained by a rotor located at the Right Atrial Appendage (RAA). Phase map obtained at the actual position provided a stable phase singularity at the RAA (Panel A). In contrast, the phase distribution at the initial iteration was irregular and no stable phase singularity could be identified at the rotor position, although other phase singularities appeared at different locations. The phase map obtained at the final iteration presented the same features as the actual one: a phase singularity near the RAA and a smooth propagation towards the rest of the atrial tissue.

A summary of all the phase singularities detected for each episode is shown at the right column of Figure 5 as a reentrant activity probability map. As expected, this reentrant activity map at the original and final position showed the highest value at the RAA (in red), where the simulated rotor 
was located, and with a Euclidean distance between maximal histogram nodes of $0.4 \mathrm{~cm}$. The reentrant activity map at the initial position did not have a maximal contribution close to the RAA, and its maximal value was present at the Left Inferior Pulmonary Vein (LIPV), $9.6 \mathrm{~cm}$ away from the RAA rotor.

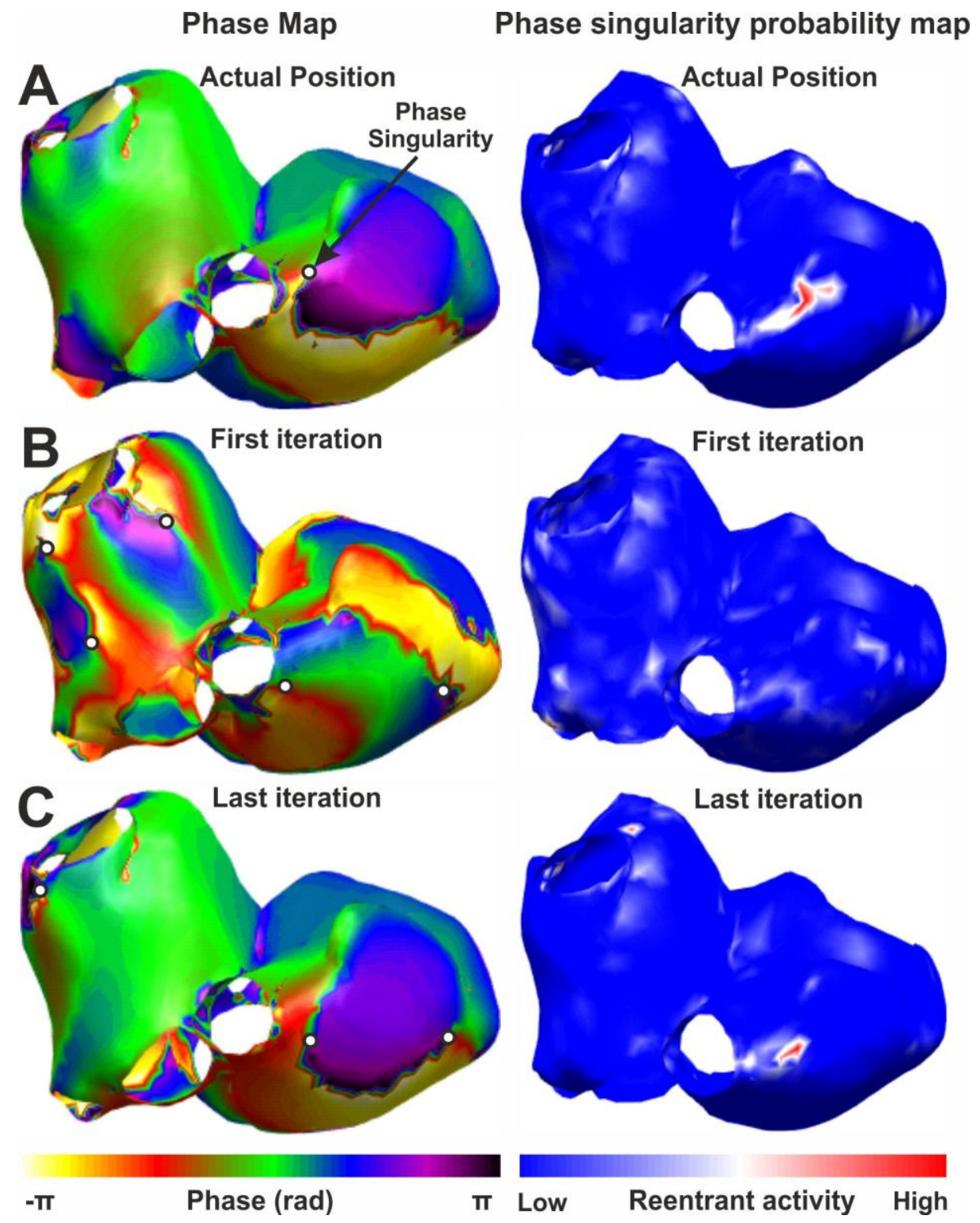

Fig. 5. Reconstruction of phase maps (left) and phase singularity probability maps (right) at the actual position $(A)$, first iteration (B) and last iteration (C). Phase singularities in the phase maps are marked with white dots.

\section{Accuracy of the AF markers after optimization}

In Figure 6 the measures of activation frequency and rotor position were compared between the actual position and the first/last iteration of the algorithm across the whole database, for both the ground-truth solution from the EGM and the ECGI reconstruction. In panel $\mathrm{A}$, the error in $\mathrm{Hz}$ between the DF values of each individual atrial node was obtained between the actual and the first/last location. When compared with the ground-truth EGM, the last position provided an error 
of $0.8 \pm 1.4 \mathrm{~Hz}$ whereas at the first position was $1.4 \pm 1.5(p<0.01)$. When compared with the ECGI solution at the actual position, the last position provided an error of $0.5 \pm 1.0 \mathrm{~Hz}$ whereas at the first position was $1.1 \pm 1.1(p<0.01)$. No significant differences were found between the errors against ECGI and EGM ground-truth at the actual position ( $p>0.05)$.

Differences in locating the reentrant activity phase maps were calculated as the distance between maximal rotor histogram sites (Figure 6.B). When compared against the ground-truth EGM distribution, the final iteration of the location algorithm provided an average distance to the original rotor location of $1.9 \pm 2.1 \mathrm{~cm}$ while at the initial position was $6.9 \pm 4.8 \mathrm{~cm}(p<0.01)$. When compared against the ECGI at the actual position, the final iteration of the location algorithm provided an average distance to the original rotor location of $1.6 \pm 1.7 \mathrm{~cm}$ while at the initial position was $7.2 \pm$ $4.0 \mathrm{~cm}(p<0.01)$, with no significant differences against the deviations provided against the groundtruth EGM solution ( $p>0.05)$.
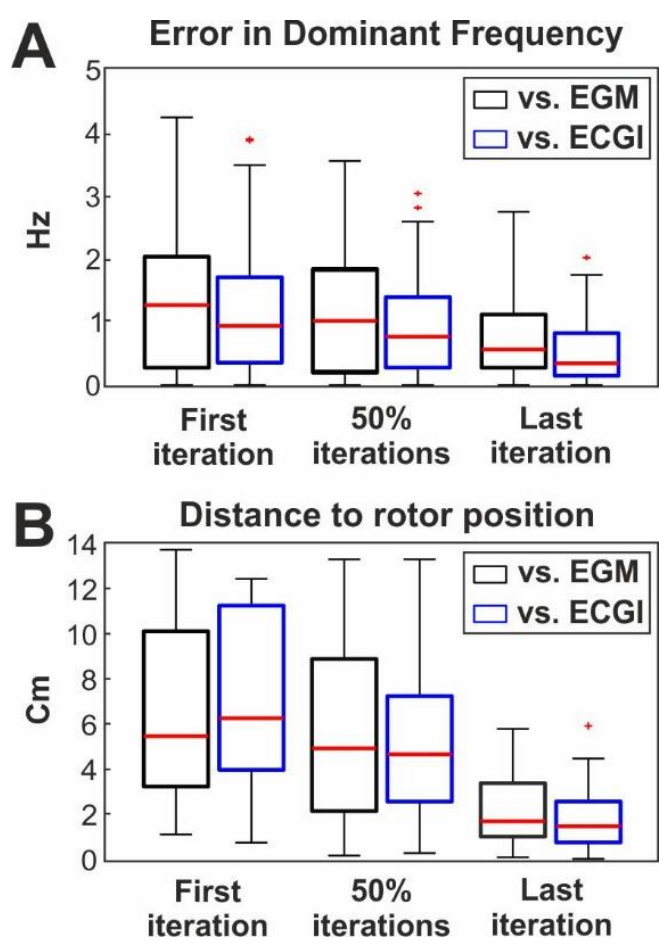

Fig. 6. Errors committed in the atrial activation rate and rotor position for the first, mid-way (50\% of iterations) and last iteration when compared with the EGM ground-truth (black) and the ECGI (blue) solutions at the actual position. 


\section{DISCUSSION}

In this study, a novel technique able to automatically locate the atrial anatomy within the torso using the curvature of the L-curve is presented. The average error obtained in atrial location was less than $0.5 \mathrm{~cm}$ for displacements and around 150 in rotation. We showed that different initial points provided similar solutions, showing the robustness of the algorithm to identify the global maximum. Moreover, we evidenced that the position provided by the location algorithm allows to properly reconstruct the main electrophysiological AF markers, such as dominant frequency gradients or reentrant activity location. On these markers we obtained an average error of $0.5 \mathrm{~Hz}$ in DF and $1.6 \mathrm{~cm}$ in the rotor position between the actual anatomical position and the final iteration of the optimization algorithm. Both measures were within the range of acceptable precision for their use in clinical practice: ablation lesions in guided procedures usually cover areas of 2 to $3 \mathrm{~cm}^{2}$ [20], and differences lower than $0.5 \mathrm{~Hz}$ are not considered as DF gradients when targeting fastest regions [17].

\section{Medical imaging acquisition in ECGI}

Medical imaging techniques such as $\mathrm{MRI}$ and $\mathrm{CT}$ play a crucial role in the identification of cardiovascular conditions that predispose to the development and perpetuation of cardiac arrhythmias. These image techniques provide important anatomical information to refine clinical strategies based on the pathological and functional information of the cardiac tissue and allows the characterization of the arrhythmogenic substrate itself. As CT and MRI are the techniques which provide the more accurate anatomical structure description and are commonly available in many health institutions, they are classically used to obtain the heart and torso models used in ECGI.

However, the use of these imaging techniques may suppose a limitation for the use of ECGI in normal clinical cardiology practice. The CT/MRI techniques are used on a huge variety of patients and medical conditions, which in some cases limit their use as screening or daily tool in the cardiovascular service due to their low priority. Therefore, CT/MRI scans are only obtained for AF patients already referred for ablation, limiting the ECGI use to this minor AF sub-population ( $<1 \%$ of AF patients). Also the need to obtain the medical images wearing the ECGI electrodes, supposes an extra logistic issue for ECGI. Nowadays there are alternative methods to MRI/CT that allow obtaining the torso geometry, as those based on photogrammetry [21]. Through this technique, Perez-Alday et al. have reported the location of 74 surface electrodes with an error in their location, compared with the typical scanner (MRI/CT) of $11.27 \pm 3.05 \mathrm{~mm}$ [22]. Schulze et al. with a similar analysis using a two calibrated cameras system and a phantom anatomical model with 80 electrodes reported an error of $1 \pm 1 \mathrm{~mm}[23]$.

On the other hand, obtaining the 3D model of the cardiac anatomy can also be performed through techniques different to MRI/CT scans. Among others, echocardiography equipment can reconstruct the cardiac surface with enough spatial resolution for ECGI. The fluoroscopy equipment or the electro-anatomic navigators present in the electrophysiology lab have also been used to obtain the cardiac anatomy for ECGI, with an error respect CT scans lower than $10 \mathrm{~mm}$ [24]. Although these are an invasive methodology (navigators) or involve patient radiation (fluoroscopy), they are commonly used in AF ablation procedures and their routine outcomes can be used to extract the atrial anatomy without a secondary scan. 


\section{Accuracy in Location and Electrical Characterization}

Reconstruction of ECGI involves the combination of electrical surface signals and the atrial and torso geometries. In both cases, the pre-processing steps involve some uncertainties or noise, which are then projected on the reconstructed maps. When different image acquisition systems are used, the co-registration of their anatomical positions has been reported to generate spatial divergences on the range of [1-8] $\mathrm{cm}$ and up to 50 when manual land-marking is used [25]. On the other hand, cardiac motion and respiration also involves spatial artifacts in the range of [1-3] cm and up to 30응 in each axis [26-27]. We opted to validate our methodology with an initial error able to mimic both conditions: normal heart movement and possible co-registration divergences.

The ability of ECGI to properly reconstruct these maps during cardiac arrhythmias in presence of spatial deviations has been extensively evaluated, and it has been reported that errors up to $1 \mathrm{~cm}$ in location can provide a proper interpretation [12, 28-29]. Different strategies have been proposed to mitigate these spatial artifacts, such as the automatic location method proposed by Coll-Font et al [30]. The results in atrial location presented in this manuscript fall within this range $(0.5 \mathrm{~cm}, 15 \mathrm{o})$, and therefore the electrophysiological features reconstructed at these locations are also within the clinically acceptable range.

The localization measures reported on this manuscript showed that higher displacement errors were committed on the vertical $(Z)$ axis than on the horizontal $(X, Y)$ axes. We hypothesize this can be provoked by the higher proximity of surface electrodes to the heart on the horizontal plane (front and back electrodes) than on the vertical plane (shoulder and hip electrodes). On the other hand, higher deviations in orientation were reported on the $Y$ axis, corresponding to the front-back axis. We believe that in this case it was provoked by the low volume displacement when rotations in the $Y$ axis occur: $Y$ axis coincides with the longest axis in the atrial anatomy (from left to right appendage), and rotations around the longer axis provoke less free space occupation/emptying. The deviations reported on this manuscript coincides also with those reported on patients on our previous work [8].

The ECGI technique also deals with the inherent and always present reconstruction error provoked by the regularization process, so even in the best scenario where no spatial deviations are present, the reconstructed electrical activity presents significant differences with the actual epicardial potentials. These reconstructions errors can be comparable and even greater than those caused by location displacements [8]. Therefore, location algorithms as the presented in this manuscript can be of great utility as long as the effect on the reconstruction provoked by the localization is lower than the intrinsic regularization errors.

\section{ECGI Reconstruction and Inverse Solution}

The solution of the inverse problem enabling to the ECGI reconstruction constitutes nowadays a challenge that many research teams are addressing. This work made use of one of the most common inverse problem methods as the Tikhonov regularization. It is considered as reference when compared to other methods although it has been shown that can over-smooth the reconstructed maps [31]. This regularization methodology was used on this manuscript as their intrinsic parameter 
(L-curve curvature) allows to refine the heart anatomy as a secondary outcome. However, the use of Tikhonov regularization on the location process do not become a limitation, once the heart is properly located, to the use of novel and more accurate inverse-problem methodologies enabling better ECGI reconstructions and/or including transmural differences [32-34].

Signal artifacts provoked by ventricular activity or their residuals after QRS subtraction can limit the accuracy of the ECGI reconstruction of the atrial electrical activity in real scenarios. However, the present optimization algorithm does not require temporal continuity of the surface signals for the ECGI reconstruction, so surface signals free of ventricular activity ( $T-Q$ intervals) can be identified and concatenated to be used by the optimization algorithm, ensuring the electrical activity under analysis is atrial activity alone. Also in cases in which patients remain in sinus rhythm, the $P$ waves can be identified and used by the optimization algorithm to refine location. This last point, as well as the use of ventricular surface activity for the ventricular activity location, should be further evaluated as the presence of a single depolarization wave-front (contrary to AF where several wavefronts can coexist) may limit the location accuracy.

This manuscript measures the accuracy of the solution provided by the location algorithm respect to the ECGI solution at the anatomical correct position as well as respect to the EGM ground truth. For both atrial frequency rate and location of reentrant activity markers, slightly higher deviations on these parameters were observed when compared against the ground truth (EGM) than when compared against the ECGI solution at the actual position, although with no statistical differences. These higher deviations are expected as the ECGI solution at the actual location has been reported to present deviations against the ground-truth [12]. However, the marker deviation provoked by the heart geometry misalignment due to co-registration or respiration is significantly greater than the inherent ECGI deviation, and therefore the presented location algorithm allows to improve the ECGI solution in these cases.

\section{Limitations}

The presented work is based on simulated data and will need human validation. The decision to use a simulated database was motivated by two main premises. First, human real data will suffer of spatial artifacts provoked by heart movement or co-registration between image and ECG position positions, and therefore the anatomical ground truth would be diluted. Second, the use of simulations from a database already calibrated against spatial artifacts [8] provides a comparative measure of the error provoked by the ECGI reconstruction and can be compared with the deviation provoked by the location algorithm.

This study used a simplistic torso model with no inhomogeneity, without no lungs nor bones, since it was previously reported that they do not significantly affect the resolution of the inverse problem $[28,35-37]$ in comparison with the effect of changes in the heart position. The inverse problem was solved using all the 771 nodes on the surface, including those at unrealistic anatomical locations for an ECG recording electrode. Previous studies suggested using these locations does not suppose a significant difference on ECGI outcome [12]. We used a unique atrial and torso anatomy and therefore the location algorithm should be validated on a representative population of cardiac patients. 
We have chosen one of the best-known methods for optimization (Pattern Search) to solve the anatomy localization, since it has provided an adequate balance between accuracy and calculation time, as one of the objectives of this project was to use this algorithm in clinical practice. However, other optimization algorithms may improve the localization results at the expense of calculation time. We also tuned the tolerance of the optimization algorithm using our simulated database, looking for a compromise between spatial accuracy and number of iterations. We found that the thresholds used here $(0.125 \mathrm{~cm} / \stackrel{\text { o }}{)}$ ) allowed to achieve this cost-effectiveness balance with a clinicalrelevant accuracy. However, these values should be reviewed in a clinical database.

\section{Conclusions}

This work presents a novel technique for identifying the heart location within the torso based on electrical measurements. Using this algorithm, we reported average errors in location less than 0.5 $\mathrm{cm}$ for displacements and around 15 degrees in rotation. The characterizations of the electrical activity by ECGI using our location algorithm was feasible for AF episodes, as the deviations in the electrophysiological markers fall within the clinically allowed: an average error less than $0.5 \mathrm{~Hz}$ for dominant frequency, and an average error of $1.6 \mathrm{~cm}$ in the reentrant activity location. This automatic location algorithm can be used to align atrial and torso anatomies from different recording techniques or to mitigate the possible spatial artifacts provoked by motion or respiration. 


\section{REFERENCES}

[1] Cuculich PS, Zhang J, Wang Y, et al. The electrophysiological cardiac ventricular substrate in patients after myocardial infarction: noninvasive characterization with electrocardiographic imaging. J Am Coll Cardiol. 2011;58(18):1893-1902. doi:10.1016/j.jacc.2011.07.029.

[2] Revishvili AS, Wissner E, Lebedev DS, et al. Validation of the mapping accuracy of a novel noninvasive epicardial and endocardial electrophysiology system. Europace. 2015;17(8):1282-1288. doi:10.1093/europace/euu339

[3] Haissaguerre $M$, Hocini $M$, Denis $A$, et al. Driver domains in persistent atrial fibrillation. Circulation. 2014;130(7):530-538. doi:10.1161/CIRCULATIONAHA.113.005421

[4] Pedrón-Torrecilla J, Rodrigo M, Climent AM, et al. Noninvasive Estimation of Epicardial Dominant High-Frequency Regions During Atrial Fibrillation. J Cardiovasc Electrophysiol. 2016;27(4):435-442. doi:10.1111/jce.12931

[5] Cuculich PS, Wang Y, Lindsay BD, et al. Noninvasive characterization of epicardial activation in humans with diverse atrial fibrillation patterns. Circulation. 2010;122(14):1364-1372. doi:10.1161/CIRCULATIONAHA.110.945709

[6] Wang Y, Schuessler RB, Damiano RJ, Woodard PK, Rudy Y. Noninvasive electrocardiographic imaging (ECGI) of scar-related atypical atrial flutter. Heart Rhythm. 2007;4(12):1565-1567. doi:10.1016/j.hrthm.2007.08.019

[7] Horácek BM, Clements JC. The inverse problem of electrocardiography: a solution in terms of single- and double-layer sources of the epicardial surface. Math Biosci. 1997;144(2):119-154. doi:10.1016/s0025-5564(97)00024-2.

[8] Rodrigo M, Climent AM, Liberos A, et al. Solving Inaccuracies in Anatomical Models for Electrocardiographic Inverse Problem Resolution by Maximizing Reconstruction Quality. IEEE Trans Med Imaging. 2018;37(3):733-740. doi:10.1109/TMI.2017.2707413

[9] Dössel O, Krueger MW, Weber FM, Wilhelms M, Seemann G. Computational modeling of the human atrial anatomy and electrophysiology. Med Biol Eng Comput. 2012;50(8):773-799. doi:10.1007/s11517-012-0924-6.

[10] Koivumäki JT, Seemann G, Maleckar MM, Tavi P. In silico screening of the key cellular remodeling targets in chronic atrial fibrillation. PLoS Comput Biol. 2014;10(5):e1003620. Published 2014 May 22. doi:10.1371/journal.pcbi.1003620.

[11] Garcia-Molla VM, Liberos A, Vidal A, et al. Adaptive step ODE algorithms for the 3D simulation of electric heart activity with graphics processing units. Comput Biol Med. 2014;44:15-26. doi:10.1016/j.compbiomed.2013.10.023.

[12] Rodrigo $M$, Climent AM, Liberos $A$, et al. Highest dominant frequency and rotor positions are robust markers of driver location during noninvasive mapping of atrial fibrillation: A computational study. Heart Rhythm. 2017;14(8):1224-1233. doi:10.1016/j.hrthm.2017.04.017. 
[13] MacLeod R.S., et al. Construction of an inhomogeneous model of the human torso for use computational electrocardiography. IEEE Eng. Med. Biol. Soc. 12(2): 688-689, 1991.

[14] Torczon V. On the convergence of pattern search algorithms. SIAM J Optim. 7(1): 1-25, 1997.

[15] Dolan E.D., et al. On the local convergence of pattern search. SIAM J Optim. 14 (2): 567-583, 2003.

[16] Rodrigo M, Guillem MS, Climent AM, et al. Body surface localization of left and right atrial high-frequency rotors in atrial fibrillation patients: a clinical-computational study. Heart Rhythm. 2014;11(9):1584-1591. doi:10.1016/j.hrthm.2014.05.013

[17] Sanders $P$, Berenfeld O, Hocini $M$, et al. Spectral analysis identifies sites of high-frequency activity maintaining atrial fibrillation in humans. Circulation. 2005;112(6):789-797. doi:10.1161/CIRCULATIONAHA.104.517011

[17] Atienza F, Almendral J, Ormaetxe JM, et al. Comparison of radiofrequency catheter ablation of drivers and circumferential pulmonary vein isolation in atrial fibrillation: a noninferiority randomized multicenter RADAR-AF trial. J Am Coll Cardiol. 2014;64(23):2455-2467. doi:10.1016/j.jacc.2014.09.053.

[18] Narayan SM, Krummen DE, Clopton P, Shivkumar K, Miller JM. Direct or coincidental elimination of stable rotors or focal sources may explain successful atrial fibrillation ablation: ontreatment analysis of the CONFIRM trial (Conventional ablation for AF with or without focal impulse and rotor modulation). J Am Coll Cardiol. 2013;62(2):138-147. doi:10.1016/j.jacc.2013.03.021.

[19] Rodrigo M, Climent AM, Liberos A, et al. Technical Considerations on Phase Mapping for Identification of Atrial Reentrant Activity in Direct- and Inverse-Computed Electrograms. Circ Arrhythm Electrophysiol. 2017;10(9):e005008. doi:10.1161/CIRCEP.117.005008.

[20] Miller JM, Kalra V, Das MK, et al. Clinical Benefit of Ablating Localized Sources for Human Atrial Fibrillation: The Indiana University FIRM Registry. J Am Coll Cardiol. 2017;69(10):1247-1256. doi:10.1016/j.jacc.2016.11.079

[21] Alioui S, Kastelein M, van Dam EM, van Dam PM. Automatic Registration of 3D Camera Recording to Model for Leads Localization. Computing in Cardiology 2017; VOL 44; ISSN: 2325-887X DOI:10.22489/CinC.2017.298-171

[22] Perez-Alday EA, Thomas JA, Kabir M, et al. Torso geometry reconstruction and body surface electrode localization using three-dimensional photography. J Electrocardiol. 2018;51(1):60-67. doi:10.1016/j.jelectrocard.2017.08.035.

[23] Schulze WH, Mackens P, Potyagaylo D, et al. Automatic camera-based identification and 3-D reconstruction of electrode positions in electrocardiographic imaging. Biomed Tech (Berl). 2014;59(6):515-528. doi:10.1515/bmt-2014-0018

[24] Ghanem RN, Ramanathan C, Jia P, Rudy Y. Heart-surface reconstruction and ECG electrodes localization using fluoroscopy, epipolar geometry and stereovision: application to noninvasive imaging of cardiac electrical activity. IEEE Trans Med Imaging. 2003;22(10):1307-1318. doi:10.1109/TMI.2003.818263. 
[25] Lee J, Thornhill RE, Nery P, et al. Left atrial imaging and registration of fibrosis with conduction voltages using LGE-MRI and electroanatomical mapping. Comput Biol Med. 2019;111:103341. doi:10.1016/j.compbiomed.2019.103341

[26] Weiss E, Wijesooriya K, Dill SV, Keall PJ. Tumor and normal tissue motion in the thorax during respiration: Analysis of volumetric and positional variations using 4D CT. Int J Radiat Oncol Biol Phys. 2007;67(1):296-307. doi:10.1016/j.ijrobp.2006.09.009

[27] Wikström K, Isacsson U, Nilsson K, Ahnesjö A. Reproducibility of heart and thoracic wall position in repeated deep inspiration breath holds for radiotherapy of left-sided breast cancer patients. Acta Oncol. 2018;57(10):1318-1324. doi:10.1080/0284186X.2018.1490027

[28] Messinger-Rapport BJ, Rudy Y. The inverse problem in electrocardiography: a model study of the effects of geometry and conductivity parameters on the reconstruction of epicardial potentials. IEEE Trans Biomed Eng. 1986;33(7):667-676. doi:10.1109/tbme.1986.325756.

[29] Messinger-Rapport BJ, Rudy Y. Noninvasive recovery of epicardial potentials in a realistic heart-torso geometry. Normal sinus rhythm. Circ Res. 1990;66(4):1023-1039. doi:10.1161/01.res.66.4.1023.

[30] Coll-Font J, Brooks DH. Tracking the Position of the Heart From Body Surface Potential Maps and Electrograms. Front Physiol. 2018;9:1727. Published 2018 Dec 3. doi:10.3389/fphys.2018.01727

[31] Figuera C, Suárez-Gutiérrez V, Hernández-Romero I, et al. Regularization Techniques for ECG Imaging during Atrial Fibrillation: A Computational Study [published correction appears in Front Physiol. 2016 Nov 17;7:556]. Front Physiol. 2016;7:466. Published 2016 Oct 14. doi:10.3389/fphys.2016.00466

[32] van der Waal J, Meijborg V, Schuler S, Coronel R, Oostendorp T. In silico validation of electrocardiographic imaging to reconstruct the endocardial and epicardial repolarization pattern using the equivalent dipole layer source model. Med Biol Eng Comput. 2020;58(8):1739-1749. doi:10.1007/s11517-020-02203-y.

[33] Chamorro-Servent J, Dubois R, Coudière Y. Considering New Regularization Parameter-Choice Techniques for the Tikhonov Method to Improve the Accuracy of Electrocardiographic Imaging. Front Physiol. 2019;10:273. Published 2019 Mar 27. doi:10.3389/fphys.2019.00273

[34] Misra S, van Dam P, Chrispin J, et al. Initial validation of a novel ECGI system for localization of premature ventricular contractions and ventricular tachycardia in structurally normal and abnormal hearts. J Electrocardiol. 2018;51(5):801-808. doi:10.1016/j.jelectrocard.2018.05.018

[35] Ramanathan C, Rudy Y. Electrocardiographic imaging: II. Effect of torso inhomogeneities on noninvasive reconstruction of epicardial potentials, electrograms, and isochrones. J Cardiovasc Electrophysiol. 2001;12(2):241-252. doi:10.1046/j.1540-8167.2001.00241.x.

[36] van Dam PM, van Oosterom A. Volume conductor effects involved in the genesis of the P wave. Europace. 2005;7 Suppl 2:30-38. doi:10.1016/j.eupc.2005.03.013. 
[37] Cheng LK, Bodley JM, Pullan AJ. Effects of experimental and modeling errors on electrocardiographic inverse formulations. IEEE Trans Biomed Eng. 2003;50(1):23-32. doi:10.1109/TBME.2002.807325. 